OPEN ACCESS

Edited by:

Pingli Lu,

Fudan University, China

Reviewed by:

Qi-Jun Chen,

China Agricultural University, China

Peng-Cheng Wei,

Anhui Academy of Agricultural

Sciences, China

Jinggao Liu,

Agricultural Research Service (USDA),

United States

${ }^{*}$ Correspondence:

Chunpeng Song

songcp@henu.edu.cn

tThese authors have contributed equally to this work.

Specialty section:

This article was submitted to Technical Advances in Plant Science,

a section of the journal

Frontiers in Plant Science

Received: 26 June 2017

Accepted: 21 July 2017

Published: 03 August 2017

Citation:

Gao W, Long L, Tian X, XU F, LiU J,

Singh PK, Botella JR and Song $C$ (2017) Genome Editing in Cotton with the CRISPR/Cas9 System.

Front. Plant Sci. 8:1364 doi: 10.3389/fpls.2017.01364

\section{Genome Editing in Cotton with the CRISPR/Cas9 System}

\author{
Wei Gao ${ }^{1 \dagger}$, Lu Long ${ }^{1 \dagger}$, Xinquan Tian', Fuchun Xu' ${ }^{1}$, Ji Liü, Prashant K. Singh', \\ Jose R. Botella ${ }^{3}$ and Chunpeng Song ${ }^{1 *}$
}

'State Key Laboratory of Cotton Biology, Henan Key Laboratory of Plant Stress Biology, School of Life Sciences, Henan University, Kaifeng, China, ${ }^{2}$ State Key Laboratory of Cotton Biology, Institute of Cotton Research of Chinese Academy of Agricultural Sciences, Anyang, China, ${ }^{3}$ School of Agriculture and Food Sciences, University of Queensland, Brisbane, QLD, Australia

Genome editing is an important tool for gene functional studies as well as crop improvement. The recent development of the CRISPR/Cas9 system using single guide RNA molecules (sgRNAs) to direct precise double strand breaks in the genome has the potential to revolutionize agriculture. Unfortunately, not all sgRNAs are equally efficient and it is difficult to predict their efficiency by bioinformatics. In crops such as cotton (Gossypium hirsutum L.), with labor-intensive and lengthy transformation procedures, it is essential to minimize the risk of using an ineffective sgRNA that could result in the production of transgenic plants without the desired CRISPR-induced mutations. In this study, we have developed a fast and efficient method to validate the functionality of sgRNAs in cotton using a transient expression system. We have used this method to validate target sites for three different genes GhPDS, GhCLA1, and GhEF1 and analyzed the nature of the CRISPR/Cas9-induced mutations. In our experiments, the most frequent type of mutations observed in cotton cotyledons were deletions ( $64 \%)$. We prove that the CRISPR/Cas9 system can effectively produce mutations in homeologous cotton genes, an important requisite in this allotetraploid crop. We also show that multiple gene targeting can be achieved in cotton with the simultaneous expression of several sgRNAs and have generated mutations in GhPDS and GhEF1 at two target sites. Additionally, we have used the CRISPR/Cas9 system to produce targeted gene fragment deletions in the GhPDS locus. Finally, we obtained transgenic cotton plants containing CRISPR/Cas9-induced gene editing mutations in the GhCLA1 gene. The mutation efficiency was very high, with $80.6 \%$ of the transgenic lines containing mutations in the GhCLA1 target site resulting in an intense albino phenotype due to interference with chloroplast biogenesis.

Keywords: cotton, CRISPR/Cas9, deletion, genome editing, insertion, mutagenesis, transient transform

\section{INTRODUCTION}

The post-genomic era confronted researchers with the need to develop efficient tools for gene function studies (Zhang F. et al., 2014; Liu et al., 2015). Reverse genetics approaches such as gene silencing have been widely used by the scientific community to elucidate gene function and decipher regulatory mechanisms and metabolic pathways (Carroll, 2011; Jinek et al., 2012; Sun et al., 2016). However, some of the available gene silencing technologies such as RNA interference 
have a number of inherent shortcomings, such as stability and incomplete silencing that can complicate the interpretation of the resulting phenotypes (Boettcher and McManus, 2015; Xu et al., 2016). Targeted genome editing has a number of advantages over other approaches as it introduces mutations in the genome that are intrinsically stable and heritable over many generations (Hilscher et al., 2017).

Diverse approaches have been developed to accomplish targetspecific genome editing, such as Zinc Finger Nucleases (ZFNs), Transcription Activator Like Effector Nucleases (TALENs), and Clustered Regularly Interspaced Short Palindromic Repeats (CRISPR)/CRISPR associated (Cas) protein systems (Gaj et al., 2013; Zhang et al., 2017). Despite their clear advantages over other approaches, ZFNs and TALENs were not widely adopted by the scientific community due to their technical complexity in the design and cloning of the molecular cassettes needed for the expression and targeting of the nuclease. The recent discovery of the CRISPR/Cas9 system has revolutionized the field as it uses a short RNA molecule to recognize the target site instead of the large polypeptides needed by ZFNs and TALENs. CRISPR/Cas9 has been rapidly adopted as the preferred genome editing tools and has been widely used in animal and plants due to its versatility, design simplicity, low cost and high efficiency (Shan et al., 2014; Wu et al., 2014; Bortesi and Fischer, 2015; Gao et al., 2015). The efficiency of sgRNAs directly affects the effective application of CRISPR/Cas9 in plants. Based on bioinformatics analysis, selection of targeted sites, and prediction of secondary structures were used to imporve the efficiency of sgRNAs (Ma et al., 2015). Protoplast transformation is also used for sgRNAs validation. However, protoplast isolation and transformation in some species are difficult to implement. There remains a need for a rapid, simple and efficient sgRNA selection method to develop CRISPR/Cas9 system.

Cotton is one of the most important fibers, a good source for biofuel production and an oil crop (Wang et al., 2012; Shi et al., 2014; Oliveira et al., 2016). Increased sequence availability has emphasized the need for rapid and cost-effective tools to create targeted mutations in order to perform much needed large-scale gene functional studies in cotton (Wang et al., 2010, 2012; Li et al., 2015; Zhang et al., 2015). CRISPR/Cas9 has been successfully used for gene editing in important crops and model systems such as rice, wheat, Arabidopsis, Nicotiana, Sorghum, poplar, maize, and tomato (Li et al., 2013; Nekrasov et al., 2013; Brooks et al., 2014; Fauser et al., 2014; Liang et al., 2014; Shan et al., 2014; Xu et al., 2014; Zhang H. et al., 2014; Fan et al., 2015; Mao et al., 2016). A growing number of agronomically useful genes are being identified in cotton, mostly involved in stress resistance and fiber development, and it is becoming urgent to develop a working CRISPR/Cas9 system for this crop (Jin and Liu, 2008; Taliercio et al., 2010; Gao et al., 2011, 2016b; Pan, 2013; Long et al., 2014). Recently, several reports have described the application of CRISPR/Cas9 in cotton targeting MYB25, GFP, GhVP, GhCLA1, or GhARG for genome editing, suggesting that CRISPR/Cas9 can be effectively used for cotton genome editing (Chen et al., 2017; Janga et al., 2017; Li C. et al., 2017; Wang P. et al., 2017; Wang Y. et al., 2017). However, the long and technically challenging transformation method for this crop limit the wide application of this technology.

In this study, we developed a fast method to experimentally validate $\operatorname{sgRNAs}$ for CRISPR/Cas9 gene editing in cotton using transient expression in cotyledon, which can be accomplished in 3 days. The new method was successfully used for multiple purposes, including validation of $s g R N A s$ targeting individual genes (GhPDS, GhCLA1, and GhEF1), simultaneous editing of homeologous genes in the cotton polyploid genome and genomic fragment deletions. Moreover, CRISPR/Cas9-induced mutations were produced in stably transformed cotton plants targeting the GhCLA1 resulting in typical albino phenotypes in the regenerated plants.

\section{MATERIALS AND METHODS}

\section{Plant Materials and Growth Conditions}

Cotton (Gossypium hirsutum L. cv. 'TM-1') seeds were imbibed in deionized water for $8 \mathrm{~h}$ before being allowed to germinate at $28^{\circ} \mathrm{C}$ for $24 \mathrm{~h}$ in the dark. Following germination, the seedlings were grown in soil at $22 / 25^{\circ} \mathrm{C}$ (night/day) under $12 \mathrm{~h} / 12 \mathrm{~h}$ light/dark conditions. Ten-day-old seedlings were used for transient transformation experiments. G. hirsutum L. cv. 'YZ-1' was used for stable transformation.

\section{Construction of CRISPR/Cas9 Vectors for Targeted Gene Editing}

We used the pYLCRIPSR/Cas9 multiplex binary vector system containing a plant codon optimized Cas9 gene (Ma et al., 2015). To confirm target specificity in the genome of cotton during target site selection, a BLAST search was conducted using the cotton genome database ${ }^{1}$. The more detailed procedure was performed as previously described (Ma et al., 2015). Subsequently, the candidate $s g R N A$ sequences were subjected to secondary structure analysis using an RNA folding platform ${ }^{2}$. Overlapping PCR was conducted to amplify the targeted sequences for the $s g R N A s$, including the two promoters utilized in this study (AtU6-29 and or AtU3b) using the primers listed in Supplementary Table S1. PCR products containing the targeted sequences were ligated into the CRISPR/Cas9 expression cassette via Golden Gate cloning, and more details were described in a previous report (Ma et al., 2015).

\section{Transient Expression and Stable Transformation}

CRISPR/Cas9 vectors were transferred into Agrobacterium tumefaciens (GV3101 and LBA4404) for transient and stable transformation experiments, respectively. For transient transformation, GV3101 was infiltrated into cotyledons of 10-day-old cotton seedlings using a needless syringe. The seedlings were incubated in a plant growth chamber at $25^{\circ} \mathrm{C}$. After incubation for $48 \mathrm{~h}$, the infiltrated cotyledons were

\footnotetext{
${ }^{1}$ http://mascotton.njau.edu.cn/html/Data/Genomefhsequence/1.html

${ }^{2}$ http://unafold.rna.albany.edu/?q=mfold/RNA-Folding-Form 2.3
} 
harvested for genomic DNA isolation and PCR/RE analysis (Gao et al., 2011). Stable transformation was performed in G. hirsutum L. cv. 'YZ-1' with LBA4404 as previously described (Jin et al., 2005).

\section{Detection of Genome Modifications}

Genomic DNA was isolated from infiltrated cotyledons using a DNA extraction kit (Tiangen Biotech, Beijing). PCR was used to amplify a genomic fragment containing the target site and appropriate restriction enzymes used to digest PCR products in order to confirm mutations at the target site. The PCR amplicons were also cloned into a TA-cloning vector and sequenced (Sangon Biotech, Shanghai).

\section{RT-PCR Analysis}

Total RNA was isolated from transgenic cotton lines using the Aidlab RNA extraction kit (Aidlab Biotechnologies, China). First-strand cDNA was performed from $1 \mu \mathrm{g}$ of total RNA using the M-MLV Reverse Transcription System (Promega, United States). PCR was performed as follows: $95^{\circ} \mathrm{C}$ for 5 min, followed by 28 cycles of amplification $\left(95^{\circ} \mathrm{C}\right.$ for $10 \mathrm{~s}, 57^{\circ} \mathrm{C}$ for $30 \mathrm{~s}$, and $72^{\circ} \mathrm{C}$ for $30 \mathrm{~s}$ ), and a final incubation at $72^{\circ} \mathrm{C}$ for $7 \mathrm{~min}$. The cotton gene ubiquitin 7 (GhUB7, Accession: DQ116441) was amplified as internal control.

\section{RESULTS}

\section{Strategy for Fast Target Validation in Cotton Cotyledons}

The available cotton transformation methods are technically demanding and time-consuming with an average of 10 months to produce $T_{0}$ transgenic lines (Jin et al., 2005). Given the length and difficulty involved in obtaining stable cotton transgenic lines, it was critical to develop a fast method to test the efficiency of the $\operatorname{sgRNAs}$. We therefore designed a fast $\operatorname{sgRNA}$ validation method for our research (Figure 1). Transient expression was achieved by infiltrating 10-day-old cotton cotyledons with $A$. tumefaciens harboring the appropriate CRISPR/Cas9 vectors (Figure 1A). The infiltrated cotyledons were harvested $48 \mathrm{~h}$ later, genomic DNA isolated and a fragment containing the target site amplified by PCR. Targeted sequences had been carefully selected to contain a restriction site in the vicinity of the PAM, therefore CRISPR-induced mutagenesis would likely destroy the enzyme recognition site. PCR amplification products were digested with the appropriate restriction enzyme and analyzed by gel electrophoresis to detect the loss of the restriction site. Finally, the gene-edited products, appearing in the electrophoresis as a high molecular size band were isolated and sequenced to re-confirm the modification and determine the nature of the CRISPR/Cas9-induced mutations (Figure 1B). This method proved to be very convenient to quickly validate the efficiency of the different $\operatorname{sgRNAs}$, being capable to simultaneously test 4-6 different targets in less than 1 week using transient expression in cotton cotyledons.

\section{CRISPR/Cas9-Induced Mutations Using Transient Expression}

In order to validate the transient transformation-based $s g R N A$ validation method we choose the elongation factor-1 protein (GhEF1) as a target (D07G1160). GhEF1 catalyzes the binding of aminoacyl-tRNAs to the A-site of the ribosome during protein synthesis. The chosen 20-bp target sequence (sgRNA1GhEF1) was located in exon \#2 of the GhEF1 gene and contained a $S t u I$ restriction site 3 bases away from the PAM for detection of mutations (Figure 2A). Transient expression with binary vectors containing expression cassettes for Cas9 alone or together with sgRNA1-GhEF1 produced PCR amplicons of approximately $0.87 \mathrm{~kb}$ in size (Figure $2 \mathrm{~B}$, lanes 1 and 2). As expected, digestion of the amplification product with $S t u \mathrm{I}$ produced two fragments of 554 and $314 \mathrm{bp}$ in the absence of target sequence sgRNA1-GhEF1 (Figure 2B, lane 3), while an extra, undigested fragment, was observed in the presence of such a sequence in the CRISPR/Cas9 cassette (Figure 2B, lanes 4-6). The undigested fragment was purified from the gel and cloned for sequence analysis. A total of 43 clones were analyzed with $63 \%$ of them harboring deletions (from 1 to 8 nucleotides in length), while $37 \%$ contained insertions (1 nucleotide) (Figure 2C). In total, 10 different mutations were detected, of which single base deletions (17/43) and single base insertions (15/43) were the most common types. Further validation of the transient transformation system was achieved using the phytoene desaturase (PDS) gene (D07G1160) (Figures 3A,B). In this case, 35 clones were sequenced revealing the presence of eight different mutation types, $57 \%$ of them deletions (from 1 to 6 nucleotides in length), and $43 \%$ insertions (1 nucleotide) (Figure 3C).

The high number of homoeologous segments present in the genome of the allotetraploid cotton results in the existence of two or more copies for most genes (Wang et al., 2014; Gao et al., 2016a). In order to obtain a functional mutant in cotton, it is imperative to edit homoeologous sequences simultaneously, and in most cases, it is possible to find highly conserved CRISPR target sequences in the homeolog genes (Gao et al., 2016a). To test whether simultaneous mutation of homoeologous genes is feasible in cotton we chose Chloroplastos alterados 1 (GhCLA1) which is involved in chloroplast development and have two homoeologous sequences (D10G1640 from the sub-genome D and A10G2292 from the sub-genome A, Figure $\mathbf{4 A}$ ). We designed a target sequence for D10G1640 that contained a single nucleotide difference at the 12th position upstream of the PAM site in A10G2292. The results of sequencing revealed successful editing events in D10G1640 as well as A10G2292, even with the imperfect target match, once more confirming the ability of CRISPR/Cas9 to edit homeologous genes in polyploids (Wang et al., 2014). As in the previous two experiments (Figures $4 \mathrm{~B}, \mathrm{C}$ ), the frequency of deletion mutations was higher (70.45\%) than insertions (27.17\%).

Combined statistical analysis of our transient expression results indicates that the most common CRISPR/Cas9-induced mutations are deletions (69.93\%) ranging from 1 to 6 nucleotides, 
A
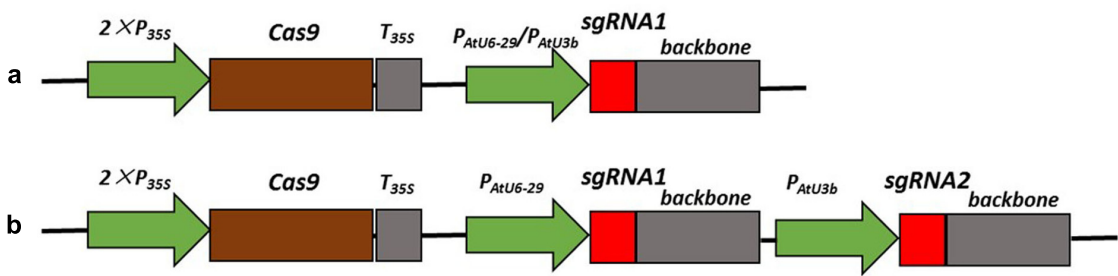

B

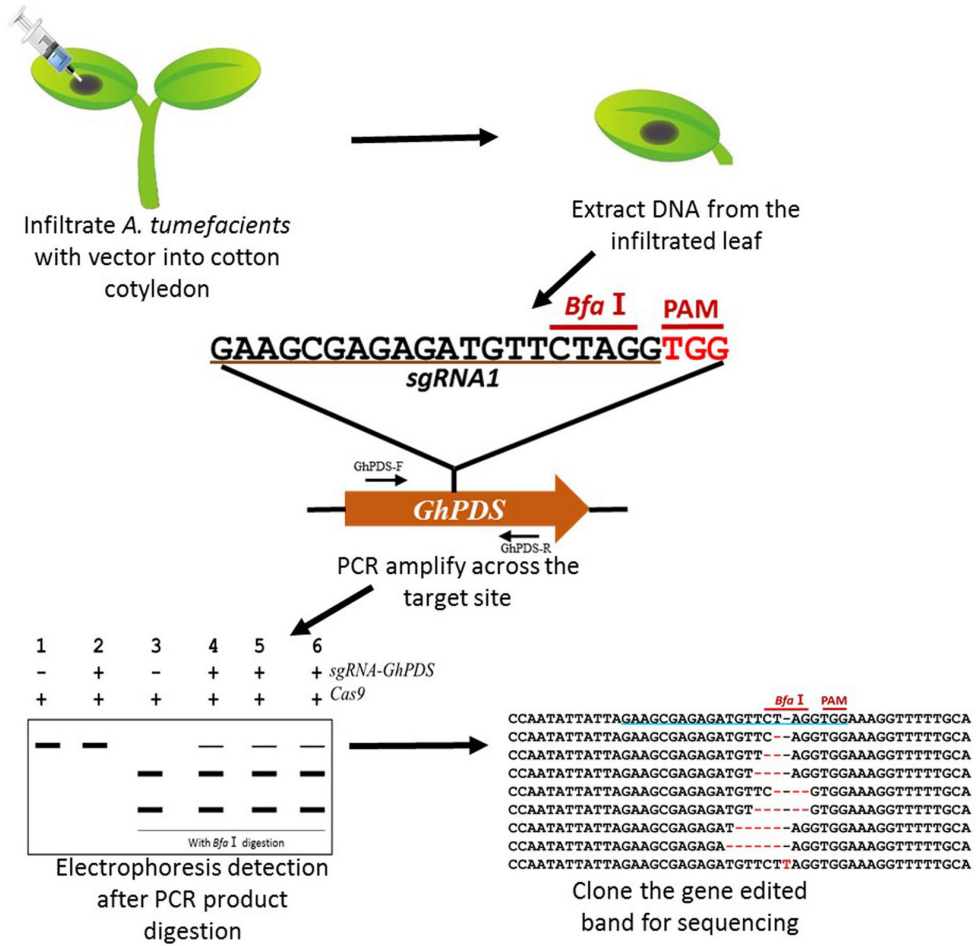

FIGURE 1 | Strategy used for the generation of the CRISPR/Cas9 system for mutagenesis in cotton via transient expression system. (A) Schematic representation of CRISPR/Cas9 vectors containing one (a) or two (b) sgRNA expression cassettes, used for transient or stable transformation in cotton. (B) Schematic description of the transient transformation method and detection of mutations.

while insertions accounted for (35.25\%), all of them being singlenucleotide insertions (Table 1). Only one instance of nucleotide replacement was detected among the 122 mutations analyzed.

\section{CRISPR/Cas9-Mediated Mutation of Two Different Genes in Transient Expression Assays}

CRISPR/Cas9 has been successfully used to create multiple mutations simultaneously (Zhang Y. et al., 2016), a feature that could prove extremely valuable for crops with lengthy and complicated transformation methods such as cotton. To validate the feasibility of creating double mutants, we used the previously described target sites for the GhEF1 and GhPDS genes (Figures 2, 3) to build two $s g R N A$ expression cassettes ( $g R R A 1$ GhEF1 and sgRNA1-GhPDS) and cloned both of them into a single CRISPR/Cas9 expression vector (Figure 5A). To minimize the probability of silencing due to the presence of repeated sequences in the transient expression cassette, expression of the GhPDS and GhEF1 sgRNAs was driven by the AtU6-29 and AtU3b promoters respectively. Transient expression of the dual $s g R N A$ vector in cotton cotyledons induced mutations in both targeted genes as indicated by the presence of a 'non-digested' amplification product for both genes (BfaI for GhPDS and StuI for GhEF1) (Figure 5B). The undigested band was purified, cloned, and subjected to sequencing analysis (Figure 5C). The sequencing results showed the presence of multiple mutation types in both genes illustrating the suitability of the CRISPR/Cas9 system for the production of double/multiple mutants for functional genomics in cotton.

\section{CRISPR/Cas9-Mediated Gene Fragment Deletion in Cotton}

Simultaneous targeting of two sites within the same gene can improve the mutagenesis efficiency of CRISPR/Cas9 and lead to the deletion of the gene fragment contained between the 
A

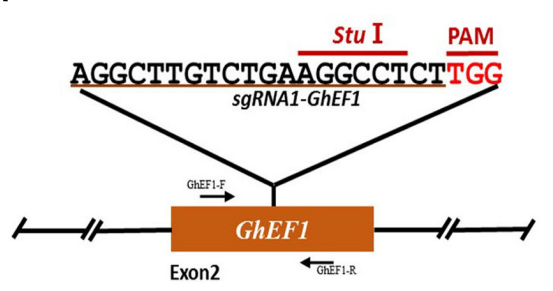

B

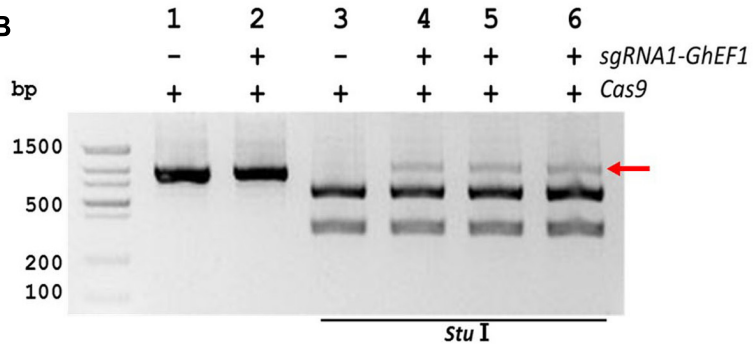

C

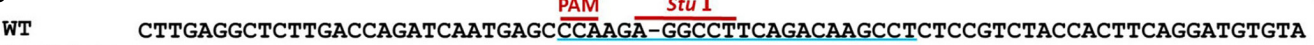
01 (2/43) CTTGAGGCTCTTGACCAGATCAATGAGCCCAAG--GGCCTTCAGACAAGCCTCTCCGTCTACCACTTCAGGATGTGTA -1 02 (15/43) CTTGAGGCTCTTGACCAGATCAATGAGCCCAAGA--GCCTTCAGACAAGCCTCTCCGTCTACCACTTCAGGATGTGTA - 1 $03(5 / 43)$ CTTGAGGCTCTTGACCAGATCAATGAGCCCAAGA---CCTTCAGACAAGCCTCTCCGTCTACCACTTCAGGATGTGTA -2 $04(1 / 43)$ СTTGAGGCTCTTGACCAGATCAATGAGCCCAAG---GCCTTCAGACAAGCCTCTCCGTCTACCACTTCAGGATGTGTA -2 $05(1 / 43)$ CTTGAGGCTCTTGACCAGATCAATGAGCCCAAGA-----TTCAGACAAGCCTCTCCGTCTACCACTTCAGGATGTGTA -4 $06(1 / 43)$ CTTGAGGCTCTTGACCAGATCAATGAGCCCAAGA-G----TCAGACAAGCCTCTCCGTCTACCACTTCAGGATGTGTA -4 $07(1 / 43)$ CTTGAGGCTCTTGACCAGATCAATGAGCCCAAGA-------CAGACAAGCCTCTCCGTCTACCACTTCAGGATGTGTA - 6 $08(1 / 43)$ CTTGAGGCTCTTGACCAGATCAATGAGC--------CСTTCAGACAAGCCTCTCCGTCTACCACTTCAGGATGTGTA -8 09 (15/43) CTTGAGGCTCTTGACCAGATCAATGAGCCCAAGATGGCCTTCAGACAAGCCTCTCCGTCTACCACTTCAGGATGTGTA +1 T $10(1 / 43)$ CTTGAGGCTCTTGACCAGATCAATGAGCCCAAGAGGGCCTTCAGACAAGCCTCTCCGTCTACCACTTCAGGATGTGTA +1 G

FIGURE 2 | CRISPR/Cas9-mediated targeted mutagenesis of GhEF1 in cotton cotyledons. (A) Target site of the sgRNA1-GhEF1 used for the transient expression experiments and relative position in the GhEF1 gene. GhEF1-F and GhEF1-R, forward and reverse primers used for amplification of the genomic fragment. (B) Detection of sgRNA1-GhEF1 targeted mutations. The gel image shows PCR amplification products from genomic DNA samples extracted after transient expression of CRISPR constructs containing Cas9 and/or the sgRNA1-GhEF1 expression cassettes. Lanes 1, 2: undigested PCR products; lanes 3-6: PCR products digested with Stul. The red arrow shows the PCR products lacking the Stul site (due to the presence of a mutation) that were subsequently purified, cloned, and analyzed by sequencing. (C) Sequencing of mutated PCR products. The target sequence (GhEF1) underlined in blue. Deletions are shown as red dashes; insertions are denoted with red letters. The frequency of each mutation is shown on the left and the mutation types on the right.

A

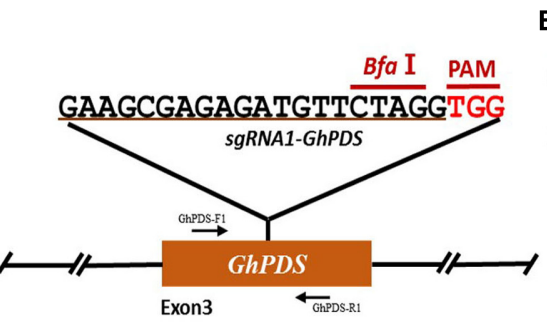

C

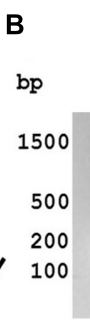

$\begin{array}{lll}1 & 2 & 3 \\ - & + & - \\ + & + & +\end{array}$

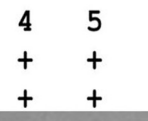

\section{6 \\ + sgRNA1-GhPDS \\ $+\operatorname{cas} 9$}

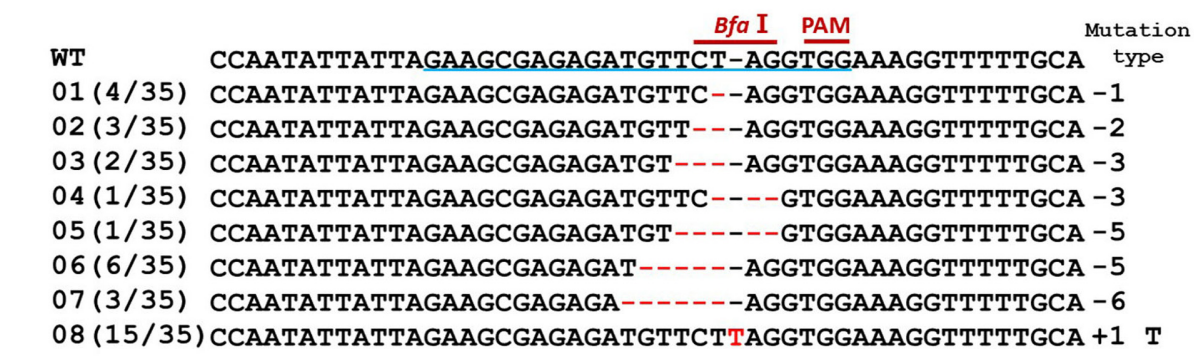

FIGURE 3 | CRISPR/Cas9-mediated targeted mutagenesis of GhPDS in cotton cotyledons. (A) Target site of the sgRNA1-GhPDS used for the transient expression experiments and relative position in the GhPDS gene. GhPDS-F1 and GhPDS-R1, forward and reverse primers used for amplification of the genomic fragment. (B) Detection of sgRNA1-GhPDS targeted mutations. The gel image shows PCR amplification products from genomic DNA samples extracted after transient expression of CRISPR constructs containing Cas9 and/or the sgRNA1-GhPDS expression cassettes. Lanes 1, 2: undigested PCR products; lanes 3-6: PCR products digested with Bfal. The red arrow shows the PCR products lacking the Bfal site (due to the presence of a mutation) that were subsequently purified, cloned, and analyzed by sequencing. (C) Sequencing of mutated PCR products. The target sequence (GhPDS) underlined in blue. Deletions are shown as red dashes, insertions are denoted with red letters. The frequency of each mutation is shown on the left and the mutation types on the right. 
A

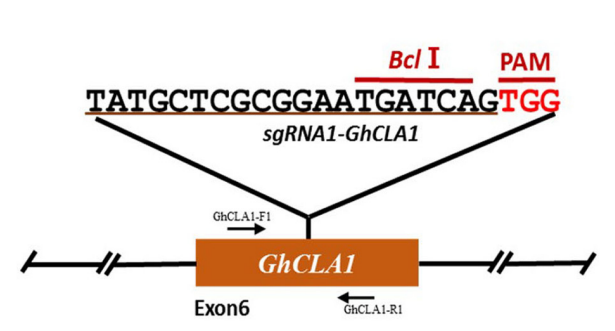

C

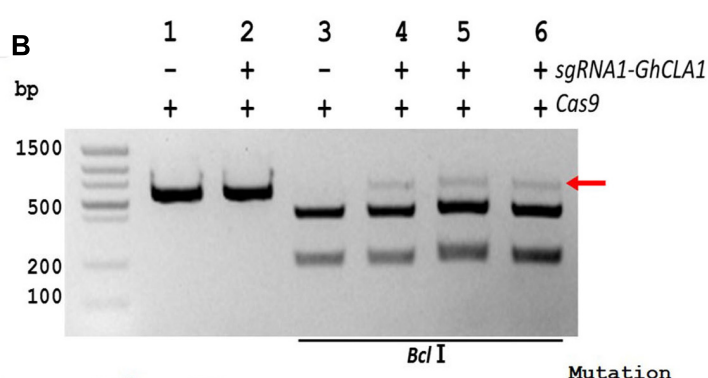

+ $B C l$ I PAM type

WT (D10G1640) TGGCTGCAAAAGTTGATGAGTATGCTCGCGGAATGAT-CA GTGGTTCTGGATCAACACTTTTCGAAG typ $01(2 / 44)$ TGGCTGCAAAAGTTGATGAGTATGCTCGCGGAATGA--CAGTGGTTCTGGATCAACACTTTTCGAAG - 1 $02(1 / 44)$ TGGCTGCAAAAGTTGATGAGTATGCTCGCGGAATG-T-CAGTGGTTCTGGATCAACACTTTTCGAAG - 1 $03(1 / 44)$ TGGCTGCAAAAGTTGATGAGTATGCTCGCGGAATGAT--AGTGGTTCTGGATCAACACTTTTCGAAG -1 $04(2 / 44)$ TGGCTGCAAAAGTTGATGAGTATGCTCGCGGAAT--T-CAGTGGTTCTGGATCAACACTTTTCGAAG -2 $05(1 / 44)$ TGGCTGCAAAAGTTGATGAGTATGCTCGCGGAATG---CAGTGGTTCTGGATCAACACTTTTCGAAG -2 $06(2 / 44)$ TGGCTGCAAAAGTTGATGAGTATGCTCGCGGAATG----AGTGGTTCTGGATCAACACTTTTCGAAG -3 $07(4 / 44)$ TGGCTGCAAAAGTTGATGAGTATGCTCGCGGAAT----CAGTGGTTCTGGATCAACACTTTTCGAAG -3 $08(3 / 44)$ TGGCTGCAAAAGTTGATGAGTATGCTCGCGGAA-----CAGTGGTTCTGGATCAACACTTTTCGAAG -4 $09(3 / 44)$ TGGCTGCAAAAGTTGATGAGTATGCTCGCGGAAT------GTGGTTCTGGATCAACACTTTTCGAAG -5 $10(1 / 44)$ TGGCTGCAAAAGTTGATGAGTATGCTCGCGGA------CAGTGGTTCTGGATCAACACTTTTCGAAG -5 $11(1 / 44)$ TGGCTGCAAAAGTTGATGAGTATGCTCGCGGAA---T---GTGGTTCTGGATCAACACTTTTCGAAG -5 $12(1 / 44)$ TGGCTGCAAAAGTTGATGAGTATGCTCGCGG-----------TTCTGGATCAACACTTTTCGAAG -12 $13(2 / 44)$ TGGCTGCAAAAGTTGATGAGTATGCTCGCGGAATGATGCAGTGGTTCTGGATCAACACTTTTCGAAG +1 G $14(6 / 44)$ TGGCTGCAAAAGTTGATGAGTATGCTCGCGGAATGATTCAGTGGTTCTGGATCAACACTTTTCGAAG +1 T WT (A10G2292) TGGCTGCAAAAGTTGATGAGTATGCTCGTGGAATGAT-CAGTGGTTCTGGATCTACACTTTTCGAAG $01(4 / 44)$ TGGCTGCAAAAGTTGATGAGTATGCTCGTGGAATGATTCAGTGGTTCTGGATCTACACTTTTCGAAG +1 T $02(4 / 44)$ TGGCTGCAAAAGTTGATGAGTATGCTCGTGGAAT----CAGTGGTTCTGGATCTACACTTTTCGAAG -3 $03(1 / 44)$ TGGCTGCAAAAGTTGATGAGTATGCTCGTGGAATGAT--AGTGGTTCTGGATCTACACTTTTCGAAG -1 $04(1 / 44)$ TGGCTGCAAAAGTTGATGAGTATGCTCGTGGAATGAT-TAGTGGTTCTGGATCTACACTTTTCGAAG C>T $05(1 / 44)$ TGGCTGCAAAAGTTGATGAGTATGCTCGTGGAATG---CAGTGGTTCTGGATCTACACTTTTCGAAG -2 $06(2 / 44)$ TGGCTGCAAAAGTTGATGAGTATGCTCGTGGAAT--------GGTTCTGGATCTACACTTTTCGAAG -7 $07(1 / 44)$ TGGCTGCAAAAGTTGATGAGTATGCTCGTG---------TGGTTCTGGATCTACACTTTTCGAAG -10

FIGURE 4 | CRISPR/Cas9-mediated targeted mutagenesis of homeologous GhCLA1 genes in cotton cotyledons. (A) Target site of the sgRNA1-GhCLA1 used for the transient expression experiments and relative position in the homeologous GhCLA1 genes (D10G1640 from D sub-genome and A10G2292 from A sub-genome). GhCLA1-F1 and GhCLA1-R1, forward and reverse primers used for amplification of the genomic fragment. (B) Detection of sgRNA1-GhCLA1 targeted mutations. The gel image shows PCR amplification products from genomic DNA samples extracted after transient expression of CRISPR constructs containing Cas9 and/or the sgRNA1-GhCLA1 expression cassettes. Lanes 1, 2: undigested PCR products; lanes 3-6: PCR products digested with Bcll. The red arrow shows the PCR products lacking the Bcll site (due to the presence of a mutation) that were subsequently purified, cloned, and analyzed by sequencing. (C) Sequencing of mutated PCR products. The target sequence (GhCLA1) is underlined in blue. Deletions are shown as red dashes, insertions are denoted with red letters. The frequency of each mutation is shown on the left and the mutation types on the right.

two targeted sites, thus increasing efficiency and providing an easier detection method (Zhang B. et al., 2016). To study whether CRISPR/Cas9 can be used for gene fragment deletion in cotton, two sgRNAs (sgRNA2-GhPDS and sgRNA3-GhPDS) were designed targeting sequences sites within the 8th exon

TABLE 1 | Determination of mutation types of transient transformation with CRISPR/Cas9 vector in cotton cotyledon.

\begin{tabular}{llccc}
\hline Mutation type & Description & $\begin{array}{c}\text { No. of clones } \\
\text { with } \\
\text { mutations }\end{array}$ & $\begin{array}{c}\text { Mutation } \\
\text { rate }\end{array}$ & $\begin{array}{c}\text { Total } \\
\text { mutation } \\
\text { rate }\end{array}$ \\
\hline Deletion & $1 \mathrm{bp}$ & 26 & $21.30 \%$ & $63.93 \%$ \\
& $2 \mathrm{bp}$ & 13 & $10.66 \%$ & \\
& $3 \mathrm{bp}$ & 13 & $10.66 \%$ & \\
Insertion & $4-6 \mathrm{bp}$ & 21 & $17.21 \%$ & \\
& $>6 \mathrm{bp}$ & 5 & $4.10 \%$ & \\
Replacement & $\mathrm{A} / \mathrm{C}$ & 40 & $32.79 \%$ & $35.25 \%$ \\
& $\mathrm{C} / \mathrm{G}$ & 3 & $2.46 \%$ & \\
& $\mathrm{C}>\mathrm{T}$ & 1 & $0.82 \%$ & $0.82 \%$
\end{tabular}

of the GhPDS gene (48 bp apart) (Figure 6A). The target site of $s g R N A 2-G h P D S$ contained an AvaI restriction site for convenient detection of the mutation. Transient expression experiments were conducted with a binary vector containing both $s g R N A$ as well as the Cas9 expression cassettes in cotton cotyledons. Genomic DNA was purified, and PCR performed using a pair of primers external to the targets to amplify a genomic fragment containing both target sites. Analysis of the PCR amplicons showed that co-expression of Cas9 and the two $s g R N A s$ resulted in the appearance of a product non-digestible with AvaI (Figure 6B). The high molecular weight bands present in lanes 4, 5, and 6 of Figure 6B were purified and re-amplified by PCR revealing the existence of two molecular species with slightly different sizes (Figure 6C; lanes 3, 4, and 5). We hypothesized that the lower molecular size amplicons were produced by deletion of the genomic fragment between the two targeted sites and were therefore purified, cloned, and sequenced. Sequence analysis of 32 clones confirmed our hypothesis showing the existence of deletion ranging from 50 to 61 nucleotides between both target sites (Figure 6D). 
A

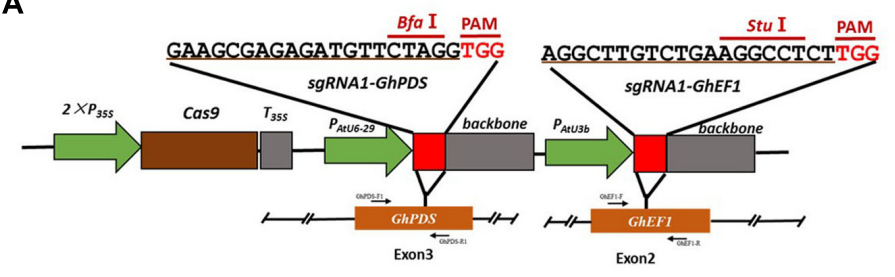

C

WT/GhPDS CCAATATTATTAGAAGCGAGAGATGTTCT-AGGTGGAAAGTTTTTGCACTA type

$01(7 / 24)$ CCAATATTATTAGAAGCGAGAGATGTTC---GGTGGAAAGGTTTTTGCACTA -2

$02(3 / 24)$ CCAATATTATTAGAAGCGAGAGAT----T-AGGTGGAAAGGTTTTTGCACTA -4

$03(3 / 24)$ CCAATATTATTAGAAGCGAGAGAT------AGGTGGAAAGGTTTTTGCACTA -5

$04(1 / 24)$ CCAATATTATTAGAAGCGAGAGAT-------GGTGGAAAGGTTTTTGCACTA - 6

$05(10 / 24)$ CCAATATTATTAGAAGCGAGAGATGTTCTTAGGTGGAAAGGTTTTTGCACTA +1 T

WT/GhEF1 CTTGAGGCTCTTGACCAGATCAATGAGC $\overline{\text { PAM }} \frac{S t u I}{\text { Mutation }}$

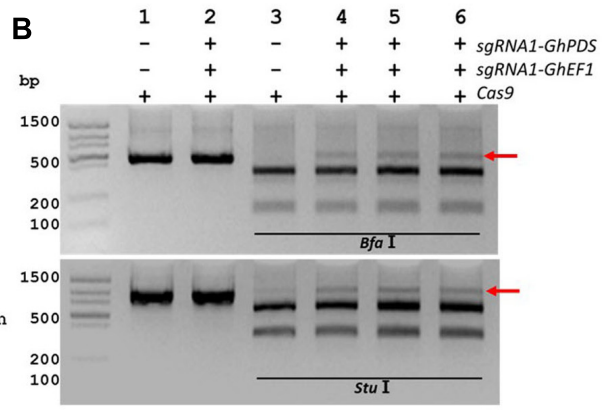
$01(8 / 18)$ CTTGAGGCTCTTGACCAGATCAATGAGCCCAAGA--GCCTTCAGACAAGCCTCTCCGTCTACCACTTCAGGATGTGTA -1 $02(4 / 18)$ CTTGAGGCTCTTGACCAGATCAATGAGCCCAAGA---CCTTCAGACAAGCCTCTCCGTCTACCACTTCAGGATGTGTA -2 $03(1 / 18)$ CTTGAGGCTCTTGACCAGATCAATGAGCCCAA------CTTCAGACAAGCCTCTCCGTCTACCACTTCAGGATGTGTA -5 $04(1 / 18)$ CTTGAGGCTCTTGACCAGATCAATGAGCCCA-----------GACAAGCCTCTCCGTCTACCACTTCAGGATGTGTA -11 $05(2 / 18)$ CTTGAGGCTCTTGACCAGATCAATGAGCCCAAGATGGCCTTCAGACAAGCCTCTCCGTCTACCACTTCAGGATGTGTA +1 T $06(2 / 18)$ CTTGAGGCTCTTGACCAGATCAATGAGCCCAAGAGGGCTTCAGACAAGCCTCTCCGTCTACCACTTCAGGATGTGA +1 G

FIGURE 5 | Simultaneous CRISPR/Cas9-mediated targeted mutagenesis of GhPDS and GhEF1 genes in cotton cotyledons. (A) Target sites of the sgRNA1-GhPDS and sgRNA1-GhEF1 used for the transient expression experiments and relative position in the GhPDS and GhEF1 gene. GhPDS-F1, GhPDS-R1, GhEF1-F and GhEF1-R, forward and reverse primers used for amplification of the genomic fragments. (B) Detection of sgRNA1-GhEF1 and sgRNA1-GhEF1 targeted mutations. The gel images show PCR amplification products from genomic DNA samples extracted after transient expression of CRISPR constructs containing Cas9 and/or the sgRNA1-GhPDS+sgRNA1-GhEF1 expression cassettes. Lanes 1, 2: undigested PCR products; lanes 3-6: PCR products digested with Bfal (upper image) or Stul (lower image). The red arrow shows the PCR products lacking the Bfal site (upper image) or Stul site (lower image) (due to the presence of a mutation) that were subsequently purified, cloned, and analyzed by sequencing. (C) Sequencing of mutated PCR products. The target sequences are underlined in blue. Deletions are shown as red dashes, insertions are denoted with red letters. The frequency of each mutation is shown on the left and the mutation types on the right.

\section{Gene Editing in Cotton by Stable Integration of CRISPR/Cas9 Cassettes}

Our developed transient transformation system was quite efficient in elucidating the suitability of CRISPR/Cas9 potential target sites in cotton cotyledons. However, this method does not produce stably mutated plants, and it is therefore not suitable for phenotypic studies. We used A.tumefaciens-mediated hypocotyl transformation to produce stable transgenic cotton lines and further confirm the feasibility of the CRISPR/Cas9 system in cotton. The GhCLA1 was selected as a target given the easily observable photobleaching phenotype caused by its inactivation (Gao et al., 2011). Some of the calli regenerated on selection medium approximately 3 months after Agrobacteriummediated transformation with the CRISPR/Cas9 binary construct showed an albino phenotype that was maintained during the entire regeneration process in stems, leaves and entire seedlings (Figures 7A,B). Ultimately, 43 putative transgenic $\mathrm{T}_{0}$ cotton lines were obtained, 36 of which tested positive for the Cas9 transgene by PCR (Figure 7C and Supplementary Figure S1). Two PCR primers flanking the sgRNA3-GhCLA1 target site in the 7th exon of GhCLA1 (Figure 8A) were used to amplify the genomic fragment in the transgenic lines. Sequence analysis of the amplification products revealed the presence of mutations in 29 of the 36 positive transgenic lines $(80.56 \%)$ (Supplementary Note S1). Unfortunately, the severe phenotypic effects caused by the inactivation of GhCLA1 (inhibition of chloroplast biogenesis), hindered the regeneration process and only some of the initial transgenic calli were successfully regenerated into plantlets, all of which showed photobleaching and severely stunted growth (Figure 8B). RTPCR analysis revealed the accumulation of Cas9 transcripts in albino mutants (Figure 8C). Six regenerated albino plantlets were analyzed for the presence of CRISPR/Cas9-induced mutations in the GhCLA1 target site. All $\mathrm{T}_{0}$ plants showed mutations in the gene with deletions $(78.90 \%)$ being more frequent than insertions $(21.1 \%)$, in agreement with our observations from the transient expression experiments (Figure 8D). Silencing of GhCLA1 using the tobacco rattle virus (TRV)-mediated virus-induced gene silencing (VIGS) also produced an albino phenotype, but this silencing method resulted in uneven and less intense photobleaching compared to the CRISPR/Cas9 mutated plants (Supplementary Figure S2), highlighting the superiority of the CRISPR/Cas9 system over RNAi-based silencing approaches.

\section{DISCUSSION}

Despite cotton's global economic importance, only a few gene functional studies were reported (Gao et al., 2011; Li et al., 2015; Zhang et al., 2015). Due to the polypoid nature of the crop, many important agronomic and quality traits controlling fiber quality, yield or defense resistance are regulated by multiple genes or genes with multiple copies, making it difficult to perform gene functional studies. Thus, most genes in the cotton genome 

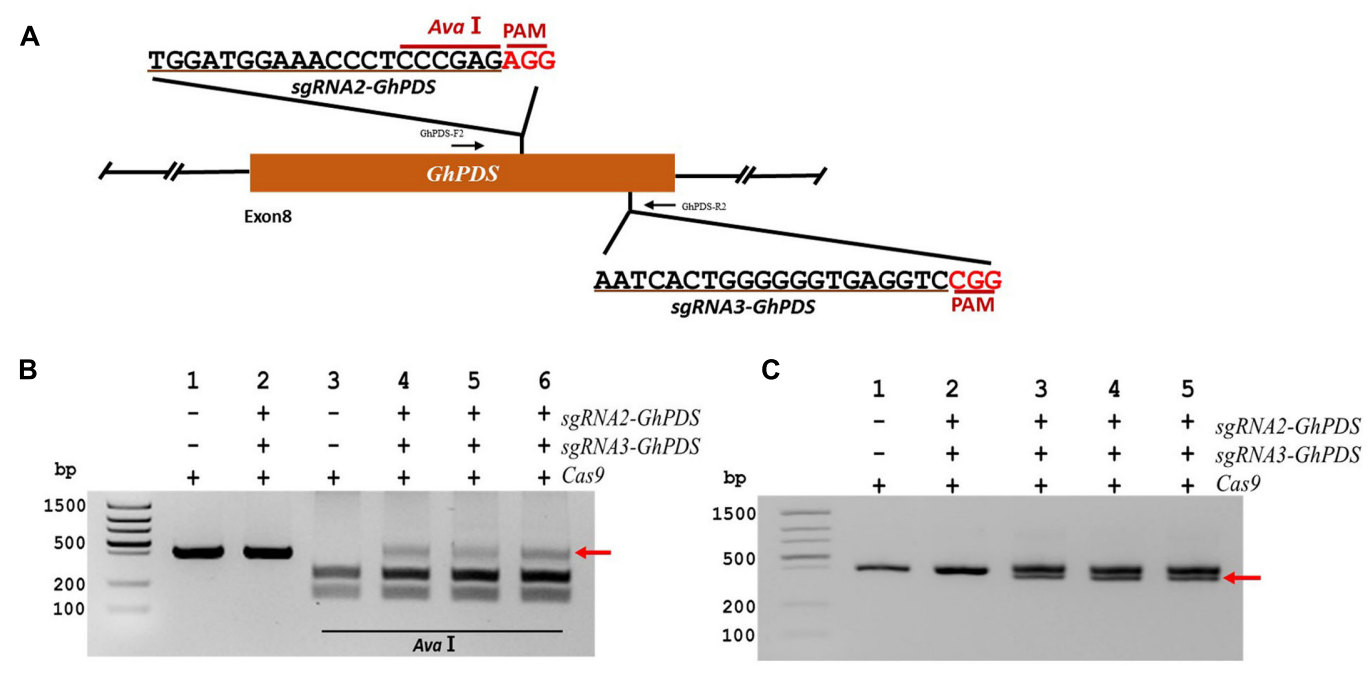

D

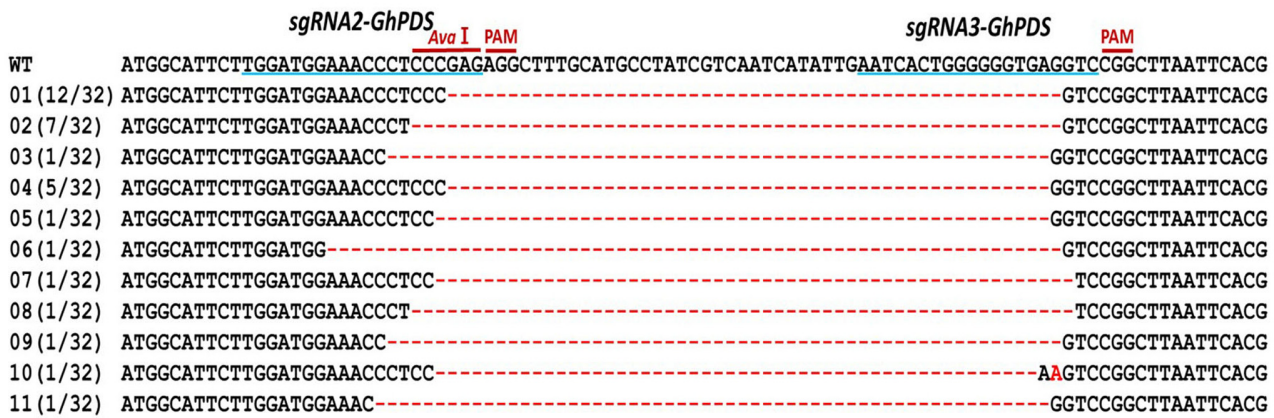

FIGURE 6 | CRISPR/Cas9-mediated targeted gene deletion in cotton cotyledons. (A) Target sites of the sgRNA2-GhPDS and sgRNA3-GhPDS used for the transient expression experiments and relative position in the GhPDS gene. GhPDS-F2 and GhPDS-R2, forward and reverse primers used for amplification of the genomic fragment. (B) Detection of sgRNA1-GhPDS targeted mutations. The gel image shows PCR amplification products from genomic DNA samples extracted after transient expression of CRISPR constructs containing Cas9 and/or the sgRNA2-GhPDS+ sgRNA3-GhPDS expression cassettes. Lanes 1, 2: undigested PCR products; lanes 3-6: PCR products digested with Aval. The red arrow shows the PCR products lacking the Bfal site (due to the presence of a mutation) that were subsequently re-amplified. (C) Gel electrophoresis of PCr amplified products from (B). The red arrow shows the smaller size PCR products (due to the presence of a deletion) that were subsequently purified, cloned, and analyzed by sequencing. (D) Sequencing of mutated PCR products from (C). The target sequences are underlined in blue. Deletions are shown as red dashes, insertions are denoted with red letters. The frequency of each mutation is shown on the left.

have no proven function, or their functional annotation has been inferred from homology to genes characterized in other plants. The advent of CRISPR/Cas9 has provided an invaluable tool for genetic studies in a large variety of plant species (Li et al., 2013; Nekrasov et al., 2013; Xu et al., 2014; Zhou et al., 2014; Fan et al., 2015; Sun et al., 2015). During the preparation of this manuscript, several reports have described the use of CRISPR/Cas9 in cotton (Chen et al., 2017; Janga et al., 2017; Li C. et al., 2017; Wang P. et al., 2017; Wang Y. et al., 2017).

The successful application of the CRISPR/Cas9 system for crop improvement or functional analyses relies on the generation of stably transformed mutants in order to perform phenotypic characterization of homozygous stable mutants. The sequence of the target site contained in the $s g R N A$ is a key factor affecting the overall mutagenesis efficiency of the CRISPR/Cas9 system as different sgRNAs can result in very different efficiencies when targeting the same gene (Ma et al., 2016). The generation of cotton mutants utilizing stable transformation is a labor-intensive and time consuming process, and therefore it is essential to select the best possible $\operatorname{sgRNA}$ in order to reduce the workload. Most of the $s g R N A$ design and selection process is currently based on bioinformatics analysis (Fan et al., 2015; Ma et al., 2016; Sun et al., 2016; Zhang B. et al., 2016). Even though bioinformatics analysis is essential to predict the specificity and theoretical efficiency of the target sites ( $\mathrm{Ma}$ et al., 2015), our work provides a fast and effective method to experimentally validate candidate sgRNAs. Based on our transient expression results targeting three different genes (GhEF1, GhPDS, and GhCLA1), the most common CRISPR/Cas9-induced mutations were deletions, which is consistent with the results reported in Arabidopsis, tobacco, rice and other species (Nekrasov et al., 2013; Li et al., 2014; Xu et al., 2014; Zhou et al., 2014; Fan et al., 2015; Sun et al., 2015). It is worth noting that the $s g R N A s$ validation process can be completed in 3 days using our transient transformation method. 


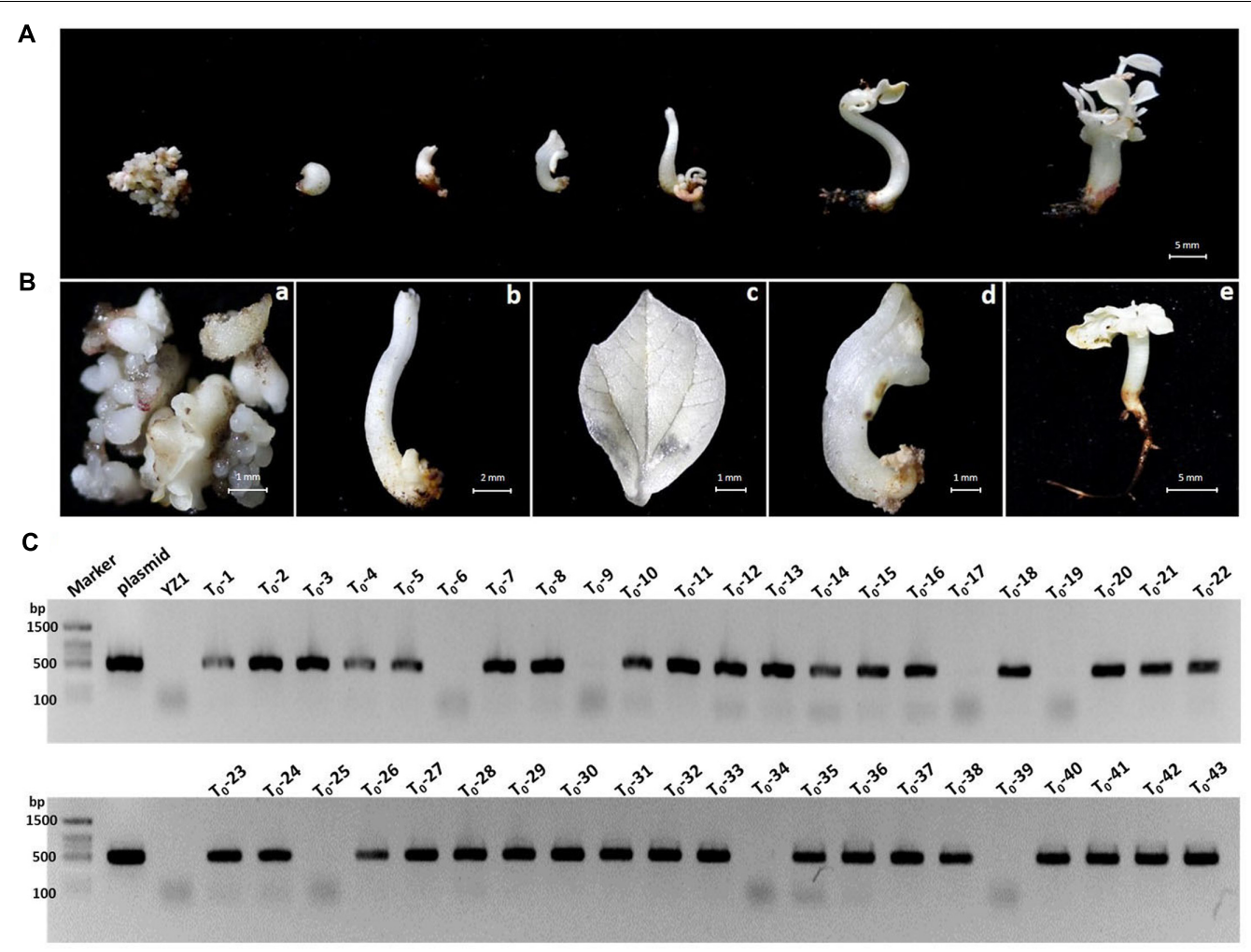

FIGURE 7 | Phenotypes of transgenic cotton tissues carrying CRISPR/Cas9 cassettes targeting the GhCLA1 gene. (A) The albino phenotype shown at different developmental stages after transformation. (B) Bleached phenotype of cotton calli (a), stem (b), leaf (c), malformed seedling (d), and normal seedling (e). (C) PCR analysis of genomic DNA isolated from 43 putative transgenic lines to detect the Cas 9 coding region. Lanes: Marker, molecular weight markers; Plasmid, positive control vector pYLCRISPR/Cas9-N; YZ-1, WT; $T_{0}-T_{43}$, independent kanamycin-resistant calli lines.

Cotton is an allotetraploid derived from hybridization and polyploidization of the $\mathrm{A}$ and $\mathrm{D}$ diploid genomes which have a high content of repetitive DNA (Li et al., 2015; Zhang et al., 2015). Thus, to perform functional studies, it is necessary to mutate multiple homoeoalleles (Wang et al., 2014). In this research, we designed a sgRNA targeting both GhCLA1 homeoalleles (D10G1640 and A10G2292) with a single-base difference in the target site and produced mutations in both alleles. In addition to single mutants, functional genomics research requires the production of double and multiple mutants in many occasions (Thung et al., 2012; Shi et al., 2016; Emonds-Alt et al., 2017; Li P. et al., 2017). Simultaneous editing of multiple genes using CRISPR/Cas9 has been reported in some plant species (Nekrasov et al., 2013; Xu et al., 2014; Fan et al., 2015; Sun et al., 2015). For example, a multiplex system targeting six of the 14 PYL families of $\mathrm{ABA}$ receptor genes was used in a single transformation experiment in Arabidopsis and homozygous sextuple mutants identified in the T3 progeny (Zhang Z. et al., 2016). We proved that simultaneous expression of two sgRNAs targeting GhPDS and GhEF1 produced mutations in both genes in cotton cotyledons. CRISPR/Cas9 can also be used to produce genomic deletions between two simultaneously targeted sites in many plant species such as Arabidopsis, rice, tobacco and wheat with deletion efficiency being higher for short fragments than long fragments
(Nekrasov et al., 2013; Fauser et al., 2014; Liang et al., 2014; Shan et al., 2014; Xing et al., 2014; Ma et al., 2015; Xie et al., 2015). In our work, deletion of 50-61 bp fragments between two target sites were detected, indicating that CRISPR/Cas9 can be applied for the deletion of chromosomal fragments in cotton.

Stably transformed cotton plants are needed for most practical applications of the CRISPR/Cas9 system, especially when studying agronomically important traits. We produced 29 $\mathrm{T}_{0}$ independent transgenic lines with CRISPR/Cas 9 constructs targeting GhCLA1 with gene editing events detected in $\sim 80 \%$ of them. This mutation frequency was similar to those previous reports in other plants despite the large size of the cotton genome (Ma et al., 2016; Zhang Y. et al., 2016). It is worth noting that the efficiency of CRISPR/Cas9 using stable transformation is very high $(\sim 80 \%)$ compared to the transient transformation. It is due to the process of stable transformation which experienced a long time tissue culture process with kanamycin resistant screening. Our data indicate that genome size does not have a significant influence on the efficiency of targeted genome mutagenesis mediated by CRISPR/Cas9 system, contrasting with the suggestion that species-specific differences in NHEJ contribute significantly to the evolution of genome size (Puchta, 2005). CRISPR/Cas9-induced mutations in GhCLA1 


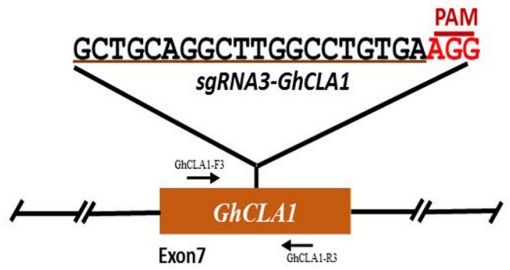

C

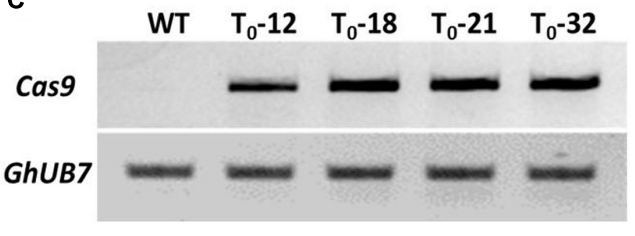

B

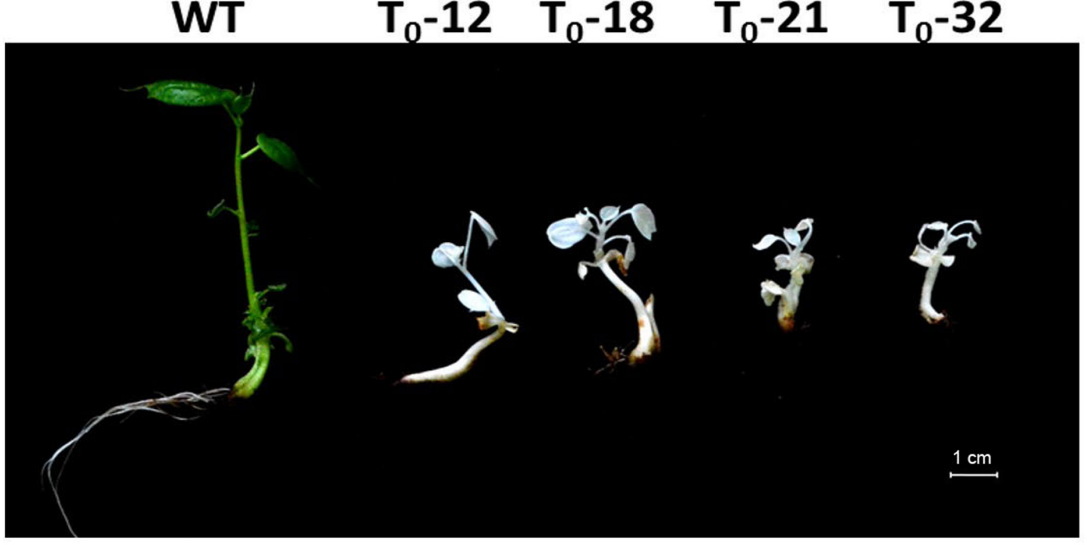

D

WT GAACAACATGCTGTCACCTTTGCTGCAGGCTTGGCCTG-TGAAGG PAMTGAAACCTTTTTGTGCAATCTACTCATCATTCAT tYP

$\mathrm{T}_{0}$-12 01 (4/22) GAACAACATGCTGTCACCTTTGCTGCAGGCTTGGCC---TGAAGGCTTGAAACCTTTTTGTGCAATCTACTCATCATTCAT -2 02 (15/22) GAACAACATGCTGTCACCTTTGCTGCAGGCTTGGCCT--TGAAGGCTTGAAACCTTTTTGTGCAATCTACTCATCATTCAT - 1 $03(3 / 22)$ GAACAACATGCTGTCACCTTTGCTGCAGGCTTGGCCTGTTGAAGGCTTGAAACCTTTTTGTGCAATCTACTCATCATTCAT +1 T

$\mathbf{T}_{0}-1801$ (9/34) GAACAACATGCTGTCACCTTTGCTGCAGGCTTGGCCT--TGAAGGCTTGAAACCTTTTTGTGCAATCTACTCATCATTCAT - 1 02 (25/34) GAACAACATGCTGTCACCTTTGCTGCAGGCTTGGCCTG---AAGGCTTGAAACCTTTTTGTGCAATCTACTCATCATTCAT -2

To-21 01(5/14) GAACAACATGCTGTCACCTTTGCTGCAGGCTTGGCC---TGAAGGCTTGAAACCTTTTTGTGCAATCTACTCATCATTCAT -2 02 (1/14) GAACAACATGCTGTCACCTTTGCTGCAGGCTTGGCC---CGAAGGCTTGAAACCTTTTTGTGCAATCTACTCATCATTCAT -2 $03(4 / 14)$ GAACAACATGCTGTCACCTTTGCTGCAGGCTTGGC----TGAATGCTTGAAACCTTTTTGTGCAATCTACTCATCATTCAT -3 G>T $04(3 / 14)$ GAACAACATGCTGTCACCTTTGCTGCAGGCTTGGCCTGCTGAAGGCTTGAAACCTTTTTGTGCAATCTACTCATCATTCAT + 1 C $05(1 / 14)$ GAACAACATGCTGTCACCTTTGCTGCAGGCTTGGCCTGATGAAGGCTTGAAACCTTTTTGTGCAATCTACTCATCATTCAT +1 A

$T_{0}-3201(2 / 13)$ GAACAACATGCTGTCACCTTTGCTGCAGGCTTGGCCT--TGAAGGCTTGAAACCTTTTTGTGCAATCTACTCATCATTCAT - 1 02 (7/13) GAACAACATGCTGTCACCTTTGCTGCAGGCTTGGCC---TGAAGGCTTGAAACCTTTTTGTGCAATCTACTCATCATTCAT -2 $03(4 / 13)$ GAACAACATGCTGTCACCTTTGCTGCAGGCTTGGC----TGAAGGCTTGAAACCTTTTTGTGCAATCTACTCATCATTCAT -3

$\mathrm{T}_{0}-3301$ (5/15) GAACAACATGCTGTCACCTTTGCTGCAGGCTTGGCCTGATGAAGGCTTGAAACCTTTTTGTGCAATCTACTCATCATTCAT + 1 A 02 (5/15) GAACAACATGCTGTCACCTTTGCTGCAGGCTTGGCCTGTTGAAGGCTTGAAACCTTTTTGTGCAATCTACTCATCATTCAT +1 T $03(4 / 15)$ GAACAACATGCTGTCACCTTTGCTGCAGGCTTGGCCT--TGAAGGCTTGAAACCTTTTTGTGCAATCTACTCATCATTCAT - 1 $04(1 / 15)$ GAACAACATGCTGTCACCTTTGCTGCAGGCTTGGCCTG---

$\mathrm{T}_{0}-3601(5 / 11)$ GAACAACATGCTGTCACCTTTGCTGCAGGCTTGGCCT--TGAAGGCTTGAAACCTTTTTGTGCAATCTACTCATCATTCAT -1 $02(4 / 11)$ GAACAACATGCTGTCACCTTTGCTGCAGGCTTGGCCTGATGAAGGCTTGAAACCTTTTTGTGCAATCTACTCATCATTCAT + 1 A $03(2 / 11)$ GAACAACATGCTGTCACCTTTGCTGCAGGCTTGGCCTGTTGAAGGCTTGAAACCTTTTTGTGCAATCTACTCATCATTCAT +1 T

FIGURE 8 | CRISPR/Cas9-mediated genome editing in transgenic cotton. (A) Target site of the sgRNA3-GhCLA1 used for the trasnformation experiments and relative position in the GhCLA1 gene. GhCLA-F3 and GhCLA-R3, forward and reverse primers used for amplification of the genomic fragment. (B) Phenotypes of cotton seedlings from wild-type plants and four $T_{0}(12,18,21$, and 32) transgenic cotton lines obtained via Agrobacterium-mediated stable transformation with a CRISPR/Cas9 construct targeting the GhCLA1 gene. (C) Electrophoresis gel showing the result of RT-PCR from total RNA isolated from WT plants and four transgenic cotton lines. GhUB7 expression was detected as internal control. (D) Targeted mutagenesis of GhCLA1. Sequencing of PCR products amplified from genomic regions containing the targeted site. Deletions are shown as red dashes, insertions are denoted with red letters. The target sequence is underlined in blue. The frequency of each mutation is shown on the left and the mutation types on the right.

produced a more obvious and uniform albino phenotype than the RNA interference induced by VIGS, emphasizing the advantage of genomic mutations over RNAi for gene silencing. The site-specific mutations created by CRISPR/Cas9 occur mostly in somatic cells and can be accurately inherited in multiple generations in Arabidopsis and rice (Ma et al., 2015). Unfortunately, the extreme phenotypic effects caused by a mutation in GhCLA1 precludes the possibility of establishing the inheritance patterns, and therefore, the heritability of CRISPR/Cas9-induced gene modifications in cotton requires further study.

In summary, our transient transformation system allows the fast validation of CRISPR/Cas9 sgRNA targets and could facilitate the adoption of high throughput functional genomic studies in cotton, especially in combination with the newly emerging viralbased CRISPR methods (Wang M. et al., 2017). 


\section{AUTHOR CONTRIBUTIONS}

WG and JB analyzed and interpreted data and wrote the manuscript. LL performed the cotton stable transformation. XT performed the transient transformation analysis. FX, JL, and PS helped to reproduce cotton material. CS designed the study and supervised all of work. All authors read and approved the final manuscript.

\section{ACKNOWLEDGMENTS}

We are grateful to Prof. Y. G. Liu (South China Agricultural University, China) for generously providing the binary pYLCRISPR/Cas9 multiplex genome targeting vector system.

\section{REFERENCES}

Boettcher, M., and McManus, M. T. (2015). Choosing the right tool for the job: RNAi, TALEN, or CRISPR. Mol. Cell. 58, 575-585. doi: 10.1016/j.molcel.2015. 04.028

Bortesi, L., and Fischer, R. (2015). The CRISPR/Cas9 system for plant genome editing and beyond. Biotechnol. Adv. 33, 41-52. doi: 10.1016/j.biotechadv.2014. 12.006

Brooks, C., Nekrasov, V., Lippman, Z. B., and Van Eck, J. (2014). Efficient gene editing in tomato in the first generation using the clustered regularly interspaced short palindromic repeats/CRISPR-associated9 system. Plant Physiol. 166, 1292-1297. doi: 10.1104/pp.114.247577

Carroll, D. (2011). Genome engineering with zinc-finger nucleases. Genetics 188, 773-782. doi: 10.1534/genetics.111.131433

Chen, X., Lu, X., Shu, N., Wang, S., Wang, J., Wang, D., et al. (2017). Targeted mutagenesis in cotton (Gossypium hirsutum L.) using the CRISPR/Cas9 system. Sci. Rep. 7:44304. doi: 10.1038/srep44304

Emonds-Alt, B., Coosemans, N., Gerards, T., Remacle, C., and Cardol, P. (2017). Isolation and characterization of mutants corresponding to the MENA, MENB, MENC and MENE enzymatic steps of 5'-monohydroxyphylloquinone biosynthesis in Chlamydomonas reinhardtii. Plant Physiol. 89, 141-154. doi: 10.1104/pp.16.01332

Fan, D., Liu, T., Li, C., Jiao, B., Li, S., Hou, Y., et al. (2015). Efficient CRISPR/Cas9mediated targeted mutagenesis in Populus in the first generation. Sci. Rep. 5:12217. doi: 10.1038/srep12217

Fauser, F., Schiml, S., and Puchta, H. (2014). Both CRISPR/Cas-based nucleases and nickases can be used efficiently for genome engineering in Arabidopsis thaliana. Plant J. 79, 348-359. doi: 10.1111/tpj.12554

Gaj, T., Gersbach, C. A., and Barbas, C. F. III. (2013). ZFN, TALEN, and CRISPR/Cas-based methods for genome engineering. Trends Biotechnol. 31, 397-405. doi: 10.1016/j.tibtech.2013.04.004

Gao, J., Wang, G., Ma, S., Xie, X., Wu, X., Zhang, X., et al. (2015). CRISPR/Cas9mediated targeted mutagenesis in Nicotiana tabacum. Plant Mol. Biol. 87, 99-110. doi: 10.1007/s11103-014-0263-0

Gao, W., Long, L., Tian, X., Jin, J., Liu, H., Zhang, H., et al. (2016a). Genomewide identification and expression analysis of stress-associated proteins (SAPs) containing A20/AN1 zinc finger in cotton. Mol. Genet. Genomics 291, 2199-2213. doi: 10.1007/s00438-016-1252-6

Gao, W., Long, L., Xu, L., Lindsey, K., Zhang, X., and Zhu, L. (2016b). Suppression of the homeobox gene HDTF1 enhances resistance to Verticillium dahliae and Botrytis cinerea in cotton. J. Integr. Plant Biol. 58, 503-513. doi: 10.1111/jipb. 12432

Gao, X., Wheeler, T., Li, Z., Kenerley, C. M., He, P., and Shan, L. (2011). Silencing GhNDR1 and GhMKK2 compromises cotton resistance to Verticillium wilt. Plant J. 66, 293-305. doi: 10.1111/j.1365-313X.2011.04491.x

Hilscher, J., Burstmayr, H., and Stoger, E. (2017). Targeted modification of plant genomes for precision crop breeding. Biotechnol. J. 12:1600173. doi: 10.1002/ biot. 201600173
The cotton seeds of G. hirsutum L. cv. 'YZ-1' used for stable transformation were kindly provided by Prof. X. L. Zhang (Huazhong Agricultural University, China). This work was financially supported by the National Natural Science Foundation of China (31601344), the Ministry of Agriculture of China (2016ZX08009-003), the National Natural Science Foundation of China (31430061), and 111 project of China.

\section{SUPPLEMENTARY MATERIAL}

The Supplementary Material for this article can be found online at: http://journal.frontiersin.org/article/10.3389/fpls.2017.01364/ full\#supplementary-material

Janga, M. R., Campbell, L. M., and Rathore, K. S. (2017). CRISPR/Cas9-mediated targeted mutagenesis in upland cotton (Gossypium hirsutum L.). Plant Mol. Biol. 94, 349-360. doi: 10.1007/s11103-017-0599-3

Jin, L. G., and Liu, J. Y. (2008). Molecular cloning, expression profile and promoter analysis of a novel ethylene responsive transcription factor gene GhERF4 from cotton (Gossypium hirstum). Plant Physiol. Biochem. 46, 46-53. doi: 10.1016/j. plaphy.2007.10.004

Jin, S., Zhang, X., Liang, S., Nie, Y., Guo, X., and Huang, C. (2005). Factors affecting transformation efficiency of embryogenic callus of Upland cotton (Gossypium hirsutum) with Agrobacterium tumefaciens. Plant Cell Tissue Organ Cult. 81, 229-237. doi: 10.1007/s11240-004-5209-9

Jinek, M., Chylinski, K., Fonfara, I., Hauer, M., Doudna, J. A., and Charpentier, E. (2012). A programmable dual-RNA-guided DNA endonuclease in adaptive bacterial immunity. Science 337, 816-821. doi: 10.1126/science.1225829

Li, C., Unver, T., and Zhang, B. (2017). A high-efficiency CRISPR/Cas9 system for targeted mutagenesis in Cotton (Gossypium hirsutum L.). Sci. Rep. 7:43902. doi: $10.1038 /$ srep 43902

Li, F., Fan, G., Lu, C., Xiao, G., Zou, C., Kohel, R. J., et al. (2015). Genome sequence of cultivated Upland cotton (Gossypium hirsutum TM-1) provides insights into genome evolution. Nat. Biotechnol. 33, 524-530. doi: 10.1038/nbt.3208

Li, J. F., Norville, J. E., Aach, J., McCormack, M., Zhang, D., Bush, J., et al. (2013). Multiplex and homologous recombination-mediated genome editing in Arabidopsis and Nicotiana benthamiana using guide RNA and Cas9. Nat. Biotechnol. 31, 688-691. doi: 10.1038/nbt.2654

Li, J. F., Zhang, D., and Sheen, J. (2014). Chapter Twenty-One - Cas9-based genome editing in Arabidopsis and tobacco. Methods Enzymol. 546, 459-472. doi: 10.1016/B978-0-12-801185-0.00022-2

Li, P., Li, Y. J., Zhang, F. J., Zhang, G. Z., Jiang, X. Y., Yu, H. M., et al. (2017). The Arabidopsis UDP-glycosyltransferases UGT79B2 and UGT79B3, contribute to cold, salt and drought stress tolerance via modulating anthocyanin accumulation. Plant J. 89, 85-103. doi: 10.1111/tpj.13352

Liang, Z., Zhang, K., Chen, K., and Gao, C. (2014). Targeted mutagenesis in Zea mays using TALENs and the CRISPR/Cas system. J. Genet. Genomics 41, 63-68. doi: 10.1016/j.jgg.2013.12.001

Liu, R., Chen, L., Jiang, Y., Zhou, Z., and Zou, G. (2015). Efficient genome editing in filamentous fungus Trichoderma reesei using the CRISPR/Cas9 system. Cell Discov. 1:15007. doi: 10.1038/celldisc.2015.7

Long, L., Gao, W., Xu, L., Liu, M., Luo, X., He, X., et al. (2014). GbMPK3, a mitogen-activated protein kinase from cotton, enhances drought and oxidative stress tolerance in tobacco. Plant Cell Tissue Organ Cult. 116, 153-162. doi: 10.1007/s11240-013-0392-1

Ma, X., Zhang, Q., Zhu, Q., Liu, W., Chen, Y., Qiu, R., et al. (2015). A robust CRISPR/Cas9 system for convenient, high-efficiency multiplex genome editing in monocot and dicot plants. Mol. Plant 8, 1274-1284. doi: 10.1016/j.molp.2015. 04.007

Ma, X., Zhu, Q., Chen, Y., and Liu, Y. G. (2016). CRISPR/Cas9 platforms for genome editing in plants: developments and applications. Mol. Plant 9, 961-974. doi: 10.1016/j.molp.2016.04.009 
Mao, Y., Zhang, Z., Feng, Z., Wei, P., Zhang, H., Botella, J. R., et al. (2016). Development of germ-line-specific CRISPR-Cas9 systems to improve the production of heritable gene modifications in Arabidopsis. Plant Biotechnol. J. 14, 519-532. doi: 10.1111/pbi.12468

Nekrasov, V., Staskawicz, B., Weigel, D., Jones, J. D., and Kamoun, S. (2013). Targeted mutagenesis in the model plant Nicotiana benthamiana using Cas 9 RNA-guided endonuclease. Nat. Biotechnol. 31, 691-693. doi: 10.1038/nbt.2655

Oliveira, M. A., Duarte, J. B., Morello, C. L., Suassuna, N. D., and Oliveira, A. B. (2016). Mixed inheritance in the genetic control of ramulosis (Colletotrichum gossypii var. cephalosporioides) resistance in cotton. Genet. Mol. Res. 15, 1-6. doi: $10.4238 /$ gmr.15038667

Pan, X. (2013). Determining gene flow in transgenic cotton. Methods Mol. Biol. 958, 261-272. doi: 10.1007/978-1-62703-212-4_21

Puchta, H. (2005). The repair of double-strand breaks in plants: mechanisms and consequences for genome evolution. J. Exp. Bot. 56, 1-14. doi: 10.1093/jxb/ eri025

Shan, Q., Wang, Y., Li, J., and Gao, C. (2014). Genome editing in rice and wheat using the CRISPR/Cas system. Nat. Protoc. 9, 2395-2410. doi: 10.1038/nprot. 2014.157

Shi, J., Chinn, M. S., and Sharma-Shivappa, R. R. (2014). Interactions between fungal growth, substrate utilization, and enzyme production during solid substrate cultivation of Phanerochaete chrysosporium on cotton stalks. Bioprocess Biosyst. Eng. 37, 2463-2473. doi: 10.1007/s00449-014-1224-3

Shi, S., Wang, T., Chen, Z., Tang, Z., Wu, Z., Salt, D. E., et al. (2016). OsHAC1;1 and OsHAC1;2 function as arsenate reductases and regulate arsenic accumulation. Plant Physiol. 172, 1708-1719. doi: 10.1104/pp.16.01332

Sun, X., Hu, Z., Chen, R., Jiang, Q., Song, G., Zhang, H., et al. (2015). Targeted mutagenesis in soybean using the CRISPR-Cas9 system. Sci. Rep. 5:10342. doi: $10.1038 /$ srep 10342

Sun, Y., Li, J., and Xia, L. (2016). Precise genome modification via sequencespecific nucleases-mediated gene targeting for crop improvement. Front. Plant Sci. 7:1928. doi: 10.3389/fpls.2016.01928

Taliercio, E., Scheffler, J., and Scheffler, B. (2010). Characterization of two cotton (Gossypium hirsutum L) invertase genes. Mol. Biol. Rep. 37, 3915-3920. doi: 10.1007/s11033-010-0048-8

Thung, L., Trusov, Y., Chakravorty, D., and Botella, J. R. (2012). Ggamma1+Ggamma2+Ggamma3=Gbeta: the search for heterotrimeric G-protein gamma subunits in Arabidopsis is over. J. Plant Physiol. 169, 542-545. doi: 10.1016/j.jplph.2011.11.010

Wang, K., Wang, Z., Li, F., Ye, W., Wang, J., Song, G., et al. (2012). The draft genome of a diploid cotton Gossypium raimondii. Nat. Genet. 44, 1098-1103. doi: 10.1038/ng.2371

Wang, M., Lu, Y., Botella, J. R., Mao, Y., Hua, K., and Zhu, J. K. (2017). Gene targeting by homology-directed repair in rice using a geminivirus-based CRISPR/Cas9 system. Mol. Plant 10, 1007-1010. doi: 10.1016/j.molp.2017. 03.002

Wang, P., Zhang, J., Sun, L., Ma, Y., Xu, J., Liang, S., et al. (2017). High efficient multisites genome editing in allotetraploid cotton (Gossypium hirsutum) using CRISPR/Cas9 system. Plant Biotechnol. J. doi: 10.1111/pbi.12755 [Epub ahead of print].

Wang, Q. Q., Liu, F., Chen, X. S., Ma, X. J., Zeng, H. Q., and Yang, Z. M. (2010). Transcriptome profiling of early developing cotton fiber by deep-sequencing reveals significantly differential expression of genes in a fuzzless/lintless mutant. Genomics 96, 369-376. doi: 10.1016/j.ygeno.2010.08.009

Wang, Y., Cheng, X., Shan, Q., Zhang, Y., Liu, J., Gao, C., et al. (2014). Simultaneous editing of three homoeoalleles in hexaploid bread wheat confers heritable resistance to powdery mildew. Nat. Biotechnol. 32, 947-951. doi: $10.1038 /$ nbt. 2969
Wang, Y., Meng, Z., Liang, C., Meng, Z., Wang, Y., Sun, G., et al. (2017). Increased lateral root formation by CRISPR/Cas9-mediated editing of arginase genes in cotton. Sci. China Life Sci. 60, 524-527. doi: 10.1007/s11427-0179031-y

Wu, X., Scott, D. A., Kriz, A. J., Chiu, A. C., Hsu, P. D., Dadon, D. B., et al. (2014). Genome-wide binding of the CRISPR endonuclease Cas9 in mammalian cells. Nat. Biotechnol. 32, 670-676. doi: 10.1038/nbt.2889

Xie, K., Minkenberg, B., and Yang, Y. (2015). Boosting CRISPR/Cas9 multiplex editing capability with the endogenous tRNA-processing system. Proc. Natl. Acad. Sci. U.S.A. 112, 3570-3575. doi: 10.1073/pnas.1420294112

Xing, H. L., Dong, L., Wang, Z. P., Zhang, H. Y., Han, C. Y., Liu, B., et al. (2014). A CRISPR/Cas9 toolkit for multiplex genome editing in plants. BMC Plant Biol. 14:327. doi: 10.1186/s12870-014-0327-y

Xu, J., Wang, X. F., Chen, P., Liu, F. T., Zheng, S. C., Ye, H., et al. (2016). RNA interference in moths: mechanisms, applications, and progress. Genes 7:88. doi: 10.3390/genes7100088

Xu, R., Li, H., Qin, R., Wang, L., Li, L., Wei, P., et al. (2014). Gene targeting using the Agrobacterium tumefaciens-mediated CRISPR-Cas system in rice. Rice 7:5. doi: 10.1186/s12284-014-0005-6

Zhang, B., Yang, X., Yang, C., Li, M., and Guo, Y. (2016). Exploiting the CRISPR/Cas9 system for targeted genome mutagenesis in Petunia. Sci. Rep. 6:20315. doi: 10.1038/srep20315

Zhang, F., Wen, Y., and Guo, X. (2014). CRISPR/Cas9 for genome editing: progress, implications and challenges. Hum. Mol. Genet. 23, R40-R46. doi: $10.1093 / \mathrm{hmg} / \mathrm{ddu} 125$

Zhang, H., Zhang, J., Wei, P., Zhang, B., Gou, F., Feng, Z., et al. (2014). The CRISPR/Cas9 system produces specific and homozygous targeted gene editing in rice in one generation. Plant Biotechnol. J. 12, 797-807. doi: 10.1111/pbi. 12200

Zhang, K., Raboanatahiry, N., Zhu, B., and Li, M. (2017). Progress in genome editing technology and its application in plants. Front. Plant Sci. 8:177. doi: $10.3389 /$ fpls.2017.00177

Zhang, T., Hu, Y., Jiang, W., Fang, L., Guan, X., Chen, J., et al. (2015). Sequencing of allotetraploid cotton (Gossypium hirsutum L. acc. TM-1) provides a resource for fiber improvement. Nat. Biotechnol. 33, 531-537. doi: 10.1038/nbt.3207

Zhang, Y., Liang, Z., Zong, Y., Wang, Y., Liu, J., Chen, K., et al. (2016). Efficient and transgene-free genome editing in wheat through transient expression of CRISPR/Cas9 DNA or RNA. Nat. Commun. 7:12617. doi: $10.1038 /$ ncomms 12617

Zhang, Z., Mao, Y., Ha, S., Liu, W., Botella, J. R., and Zhu, J. K. (2016). A multiplex CRISPR/Cas9 platform for fast and efficient editing of multiple genes in Arabidopsis. Plant Cell Rep. 35, 1519-1533. doi: 10.1007/s00299-015-1900-z

Zhou, H., Liu, B., Weeks, D. P., Spalding, M. H., and Yang, B. (2014). Large chromosomal deletions and heritable small genetic changes induced by CRISPR/Cas9 in rice. Nucleic Acids Res. 42, 10903-10914. doi: 10.1093/nar/ gku806

Conflict of Interest Statement: The authors declare that the research was conducted in the absence of any commercial or financial relationships that could be construed as a potential conflict of interest.

Copyright (C) 2017 Gao, Long, Tian, Xu, Liu, Singh, Botella and Song. This is an open-access article distributed under the terms of the Creative Commons Attribution License (CC BY). The use, distribution or reproduction in other forums is permitted, provided the original author(s) or licensor are credited and that the original publication in this journal is cited, in accordance with accepted academic practice. No use, distribution or reproduction is permitted which does not comply with these terms. 